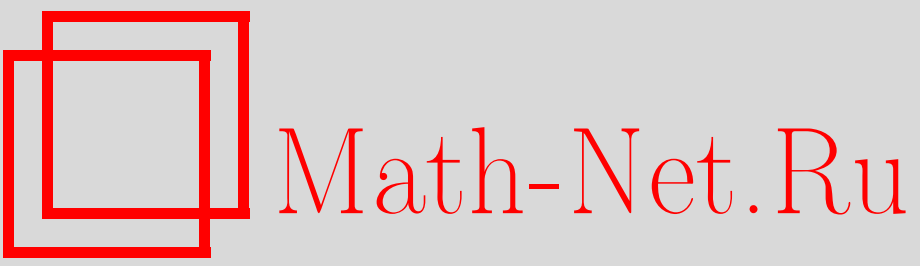

Г. И. Калмыков, Об оценке радиуса сходимости майеровских разложений (случай неотрицательного потенциала), ТМФ, 1998, том 116, номер 3, 417-430

DOI: https://doi.org/10.4213/tmf913

Использование Общероссийского математического портала Math-Net.Ru подразумевает, что вы прочитали и согласны с пользовательским соглашением http://www. mathnet.ru/rus/agreement

Параметры загрузки:

IP: 35.174 .16 .151

26 апреля 2023 г., $17: 04: 30$ 


\section{ОБ ОЦЕНКЕ РАДИУСА СХОДИМОСТИ МАЙЕРОВСКИХ РАЗЛОЖЕНИЙ (СЛУЧАЙ НЕОТРИЦАТЕЛЬНОГО ПОТЕНЦИАЛА)}

Для случая неотрицательного регулярного парного потенциала дана последовательность оценок сверху радиуса сходимости разложения термодинамического предела давления по степеням активности, сходящаяся к этому радиусу.

В статье рассматривается большой канонический ансамбль однокомпонентных систем частиц с парным взаимодействием [1], заключенных в $\nu$-мерной связной области $\Lambda \subset \mathbf{R}^{\nu}$. Предполагается, что множество $\Lambda$ ограничено и измеримо по Лебегу, а частищы взаимодействуют посредством центральных сил, характеризуемых потенциалом парного взаимодействия $\Phi(\mathbf{r})$, где $\mathbf{r}=\left(r^{(1)}, r^{(2)}, \ldots, r^{(\nu)}\right) \in \mathbf{R}^{\nu}$. Предполагается, что потенциал $\Phi(\mathbf{r})$ является измеримой функцией и удовлетворяет условию устойчивости [1]: сушествует такое число $B \geqslant 0$, что неравенство

$$
\sum_{1 \leqslant i<j \leqslant n} \Phi\left(\mathbf{r}_{i}-\mathbf{r}_{j}\right) \geqslant-n B
$$

имеет место при всех $n \geqslant 2$ и всех $\mathbf{r}_{1}, \mathbf{r}_{2}, \ldots, \mathbf{r}_{n} \in \mathbf{R}^{\nu}$. Введем следующие обозначения:

$$
(\mathbf{r})_{n}=\left(\mathbf{r}_{1}, \mathbf{r}_{2}, \ldots, \mathbf{r}_{n}\right)
$$

где $\mathbf{r}_{i} \in \mathbf{R}^{\nu}, i=1,2, \ldots, n$;

$$
\begin{gathered}
U(\mathbf{r})_{n}=U\left(\mathbf{r}_{1}, \mathbf{r}_{2}, \ldots, \mathbf{r}_{n}\right)=\sum_{1 \leqslant i<j \leqslant n} \Phi\left(\mathbf{r}_{i}-\mathbf{r}_{j}\right), \\
d(\mathbf{r})_{n}=d \mathbf{r}_{1} d \mathbf{r}_{2} \ldots d \mathbf{r}_{n},
\end{gathered}
$$

где

$$
d \mathbf{r}_{i}=d r_{i}^{(1)} d r_{i}^{(2)} \ldots d r_{i}^{(\nu)}
$$

* Московский государственный заочный институт пищевой промышленности, Центр физических исследований им. П.Н. Лебедева, Москва, Россия 
$k$ - постоянная Больцмана, $T$ - абсолютная температура, $\beta=1 / k T$ - обратная температура, $|\Lambda|$ - лебегова мера множества $\Lambda$.

Из условия (1) следует сходимость большой статистической суммы [1]

$$
\Xi(\Lambda, z, \beta)=1+z+\sum_{n=2}^{\infty} \frac{z^{n}}{n !} \int_{\Lambda^{n}} \exp \left\{-\beta U(\mathbf{r})_{n}\right\}(d \mathbf{r})_{n},
$$

где $z$ - активность $[1,2]$. При этом, как известно [2], давление

$$
p_{\Lambda}(z)=\beta^{-1}|\Lambda|^{-1} \ln \Xi(\Lambda, z, \beta)
$$

разлагается в ряд по степеням активности,

$$
p_{\Lambda}(z)=\beta^{-1}|\Lambda|^{-1} \ln \Xi(\Lambda, z, \beta)=\beta^{-1} \sum_{n=1}^{\infty} b_{n}(\Lambda, \beta) z^{n} .
$$

В дальнейшем предполагается, что парный потенциал $\Phi(\mathbf{r})$ удовлетворяет условию регулярности [1]: интеграл

$$
C(\beta)=\int_{\mathbf{R}^{\nu}}\left|e^{-\beta \Phi(\mathbf{r})}-1\right| d \mathbf{r}
$$

сходится при некотором $\beta>0$ (а следовательно, при всех $\beta>0$ ).

Если множество $\Lambda$ стремится к бесконечности в смысле Фишера [1], то в некотором круге ненулевого радиуса комплексной $z$-плоскости с центром в точке $z=0$ ряд в правой части (6) сходится почленно к пределу $[1,3]$

$$
\lim _{\Lambda \rightarrow \infty} \beta^{-1} \sum_{n=1}^{\infty} b_{n}(\Lambda, \beta) z^{n}=\beta^{-1} \sum_{n=1}^{\infty} b_{n}(\beta) z^{n} .
$$

При положительных значениях $z$, принадлежаших кругу сходимости ряда в правой части (8), определяемая этим рядом функция, представляет собой термодинамический предел давления,

$$
p(z)=\lim _{n \rightarrow \infty} p_{\Lambda}(z)=\beta^{-1} \sum_{n=1}^{\infty} b_{n}(\beta) z^{n} .
$$

Последний ряд называется майеровским разложением термодинамического предела давления.

Одной из задач классической статистической механики является $[1,4]$ определение радиуса сходимости майеровского разложения. В книге [1] получена оценка снизу этого радиуса для случая устойчивого и регулярного парного потенциала. Для случая регулярного неотришательного парного потенциала в статье [5] были найдены двусторонние оценки радиуса сходимости. Эти же оценки были получены более простым путем в статье [3]. Однако указанные оценки являются, по-видимому, очень грубыми. В связи с этим И.И. Иванчик поставил перед автором статьи задачу совершенствования этих оценок.

В данной статье для случая регулярного неотрицательного парного потенциала уточняется полученная в работе [5] оценка сверху радиуса сходимости майеровского разложения в правой части (9). Получена последовательность верхних оценок этого радиуса, сходящаяся к нему.

Основной результат статьи формулируется в следующей теореме. 
ТЕОремА. Если потенциал парного взаимодействия неотрицательньй и регулярный, то:

1) при любом $k \geqslant 2$ величина $\left(k\left|b_{k}(\beta)\right|\right)^{-1 /(k-1)}$ является оченкой сверху радиуса сходимости $R$ майеровского разложсения (9);

2) последовательность

$$
\left(2\left|b_{2}(\beta)\right|\right)^{-1},\left(3\left|b_{3}(\beta)\right|\right)^{-1 / 2}, \ldots,\left(n\left|b_{n} k(\beta)\right|\right)^{-1 /(n-1)}, \ldots
$$

имеет конечный предел, равный $R$.

Доказательство опирается на сформулированные и доказанные ниже шесть лемм и четыре следствия из них.

Предварительно мы приведем некоторые необходимые понятия, введенные в статьях $[3,6,7]$. Будем придерживаться терминологии книги [8] и статьи $[6]$.

Обозначим через $T(V)=\{t\}$ множество помеченных растуших деревьев с множеством вершин $V=\left\{v_{1}, v_{2}, \ldots, v_{n}\right\}$. Без ограничения обшности можно считать, что вершины дерева $t$ помечены натуральными числами, и отождествлять множество вершин $V$ с множеством натуральных чисел мощности $n$. В дальнейшем изложении мы будем считать, что множество $V$ представляет собой множество $n$ натуральных чисел. Если $t-$ дерево из множества $T(V)$, то множество его дуг будет обозначаться через $X(t)$. Дуга, исходяшая из вершины $i$ и входяшая в вершину $j$, отождествляется с упорядоченной парой $(i, j)$. Множество дуг дерева $t$ отождествляется с множеством соответствующих пар. Подмножество деревьев с корнем $\alpha$ обозначается $T(V, \alpha)$. Подмножество деревьев $T\left(V_{n}, 1\right)=\{t\}$, где $V_{n}=\{1,2, \ldots, n\}$ обозначается как $T_{n}=\{t\}$.

Обозначим через $V^{[2]}$ множество упорядоченных пар, определяемое равенством

$$
V^{[2]}=(V \times V) \backslash\left(\bigcup_{i \in V}\{(i, i)\}\right) .
$$

Элементы этого множества будем называть дугами.

ОПРЕДЕЛЕНИЕ 1. Две дуги из множества $V^{[2]}$ называются соседними, если у них совпадают вторые компоненты.

ОПреДЕлЕНИЕ 2. Дуга $(i, j)$ предшествует соседней дуге $(k, j)$, если $i<k$.

Пусть $V(t, h)$ - слой вершин дерева $t$, находящийся на высоте $h ; H(t)$ - высота дерева $t ;|M|$ - мощность конечного множества $M$.

$\Gamma$ раф $G$ с множеством помеченных вершин $V$ и множеством ребер $\widetilde{X}=\{\tilde{x}\}$ обозначается $G(V, \widetilde{X})$. Каждое ребро $\tilde{x} \in \widetilde{X}$ графа $G(V, \widetilde{X})$ представляет собой неупорядоченную пару $\{i, j\}$ различных вершин множества $V$. В дальнейшем каждая неупорядоченная пара $\{i, j\}$ вершин из множества $V$ будет называться ребром. Множество ребер графи $G$ обозначается через $\widetilde{X}(G)$, а множество вершин графа $G$ - через $V(G)$.

Пусть $t \in T(V, \alpha)$. Обозначим

$$
Z(t)=\bigcup_{h=1}^{H(t)}\{\tilde{x}=\{i, j\}: i, j \in V(t, h), i \neq j\} .
$$


Если $j \in V(t, h)(2 \leqslant h \leqslant H(t))$ и $(i, j)$ - дуга дерева $t$, входяшая в вершину $j$, то $Y(t, j)-$ совокупность всех дуг из множества $V^{[2]}$, исходяших из вершин слоя $V(t, h-1)$, входяших в вершину $j$ и следуюших за дугой $(i, j)$. Пусть

$$
Y(t)=\bigcup_{h=2}^{H(t)} \bigcup_{j \in V(t, h)} Y(t, j),
$$

$\widetilde{Y}(t)$ - множество всех неориентированных ребер, которые получаются из дуг множества $Y(t)$ после удаления ориентации,

$$
\tilde{X}^{\prime}(t)=\tilde{Y}(t) \cup Z(t),
$$

$\widetilde{X}(t)$ - множество ребер, которые получаются из дуг множества $X(t)$ после удаления ориентации.

ОПреДЕЛЕНиЕ 3. Максимальным надграфом дерева $t \in T(V, \alpha)$ называется граф с множеством вершин $V$ и множеством ребер $\widetilde{X}(t) \cup \widetilde{X}^{\prime}(t)$.

Как известно, всякое растушее дерево является ориентированным графом. Исходя из определения изоморфизма ориентированных помеченных графов [9] изоморфизм растуших помеченных деревьев можно сформулировать следуюшим образом.

ОПреДЕЛЕниЕ 4. Два растуших помеченных дерева $t(V, X)$ и $t^{\prime}\left(V^{\prime}, X^{\prime}\right)$ изоморфны тогда, когда сушествует такое взаимно однозначное отображение $\varphi: V \leftrightarrow V^{\prime}$, что если дуга $(u, v)$ принадлежит множеству $X$ дуг дерева $t$, то дуга $(\varphi(u), \varphi(v))$ принадлежит множеству $X^{\prime}$ дуг дерева $t^{\prime}$.

Для целей этой статьи потребуется более сильное определение изоморфизма растуших помеченных деревьев. Такой более сильньй изоморфизм мы назовем максимальным изоморфизмом.

ОПреДЕЛЕниЕ 5. Два растущих помеченных дерева $t(V, X)$ и $t^{\prime}\left(V^{\prime}, X^{\prime}\right)$ являются максимально изоморфными, если существует взаимно однозначное отображение $\varphi: V \leftrightarrow V^{\prime}$, удовлетворяюшее условиям: 1$)$ если дуга $(u, v)$ принадлежит множеству $X$ дуг дерева $t$, то дуга $(\varphi(u), \varphi(v))$ принадлежит множеству $X^{\prime}$ дуг дерева $\left.t^{\prime} ; 2\right)$ отображение $\varphi$ сохраняет смежность ребер максимального надграфа $G(t)$ дерева $t$, т.е. если $\{u, v\} \in \widetilde{X}(G(t))$, то ребро $\{\varphi(u), \varphi(v)\}$ принадлежит множеству ребер максимального надграфа $G\left(t^{\prime}\right)$ дерева $\left.t^{\prime} ; 3\right)$ обратное отображение $\varphi^{-1}: V^{\prime} \leftrightarrow V$, так же как и отображение $\varphi$, сохраняет смежность ребер максимального надграфа $G\left(t^{\prime}\right)$ дерева $t^{\prime}$, т.е. если $\left\{u^{\prime}, v^{\prime}\right\} \in \widetilde{X}(G(t))$, то ребро $\left\{\varphi^{-1}\left(u^{\prime}\right), \varphi^{-1}\left(v^{\prime}\right)\right\}$ принадлежит множеству ребер $\widetilde{X}(G(t))$ максимального надграфа $G(t)$ дерева $t$.

Рассмотрим множество деревьев $T(V, \alpha)$. Элементы множества $V$ обозначим $v_{1}, v_{2}, \ldots, v_{n}$, где $n=|V|$. Без ограничения общности можно считать, что $v_{1}=\alpha$ и $v_{i}<v_{j}$, если $2 \leqslant i<j \leqslant n$. Определим отображение $\omega$ на множестве $V$ формулой

$$
\omega\left(v_{i}\right)=i .
$$


Отображение $\omega$ устанавливает взаимно однозначное соответствие между множествами $V$ и $V_{n}$, где $n=|V|$. Оно индуцирует взаимно однозначное отображение $\Omega$ множества дуг $V^{[2]}$ на множество дуг $V_{n}^{[2]}$, определяемое формулой

$$
\Omega((u, v))=(\omega(u), \omega(v))
$$

где $(u, v) \in V^{[2]}$.

Обозначим через $A$ оператор, определенный на множестве деревьев $T(V, \alpha)$ и отображаюший дерево $t \in T(V, \alpha)$ с множеством дуг $X(t)$ в ориентированный помеченный граф̆ At с множеством вершин $V_{n}$ и множеством дуг $\Omega(X(t))$.

Лемма 1. При любом $t \in T(V, \alpha)$ граф $A t\left(V_{n}, \Omega(X(t))\right)$ является деревом. При этом расстояние любой вериинь $v \in V$ от корня в дереве $t$ равно расстоянию вериины $\omega(v)$ от корня в дереве At.

ДокаЗАтельство. Докажем, что любая вершина $j \in V_{n}$, где $j \neq 1$, достижима из вершины $1 \in V_{n}$ в графе $A t$. Пусть последовательность $\alpha, v_{i(1)}, v_{i(2)}, \ldots, v_{i(\mu)}, v_{j}$ есть путь из корня $\alpha$ в вершину $v_{j}$ в дереве $t$. Тогда дуги $\left(\alpha, v_{i(1)}\right),\left(v_{i(1)}, v_{i(2)}\right), \ldots$ $\ldots,\left(v_{i(\mu)}, v_{j}\right)$ принадлежат множеству дуг $X(t)$. При этом из определения отображения $\Omega$ следует, что дуги $(i, i(1)),(i(1), i(2)), \ldots,(i(\mu), j)$ принадлежат множеству дуг $\Omega(X(t))$. Следовательно, вершина $j$ достижима из вершины 1 в графе $A t$, а длина пути $1, i(1), i(2), \ldots, i(\mu), j$ в графе $A t$ совпадает с длиной пути $\alpha, v_{i(1)}, v_{i(2)}, \ldots, v_{i(\mu)}, v_{j}$ в дереве $t$. Так как отображение $\Omega$ является взаимно однозначным, то число дуг в множестве $\Omega(X(t))$, как и число дуг в множестве $X(t)$, равно $n-1$. Отсюда вытекает, что граф̆ $A t$ - помеченное растушее дерево. Следовательно, какова бы ни была вершина $j \in V_{n}$, в дереве $A t$ сушествует лиш один путь из корня $1 \in V_{n}$ в эту вершину. Как уже доказано, длина этого пути совпадает с длиной пути из корня $\alpha \in V$ в вершину $V_{j}$ в дереве $t$. Что и требовалось доказать.

ЛЕмма 2. При любом $t \in T(V, \alpha)$ деревья $t$ и At максимально изоморфны.

ДокАЗАТЕльство. Отображение $\omega$ является по определению взаимно однозначным отображением множества $V$ на множество $V_{n}$, где $n=|V|$. Из определения отображения $\Omega$ следует, что если $(u, v) \in X(t)$, то дуга $(\omega(u), \omega(v))$ принадлежит множеству дуг дерева At. Из леммы 1 вытекает, что

$$
\omega(V(t, h))=V(A t, h)
$$

Таким образом, отображение $\omega$ сохраняет смежность пар вершин максимального надграфа $G(t)$ дерева $t$, принадлежаших одному и тому же слою $V(t, h)$ вершин дерева $t$, т.е. если $\{u, v\} \in \widetilde{X}(G(t))$ и вершины $u$ и $v$ принадлежат одному и тому же слою $V(t, h)$ вершин дерева $t$, то ребро $\{\omega(u), \omega(v)\}$ принадлежит множеству ребер $\widetilde{X}(G(A t))$ максимального надграфа дерева $A t$.

Пусть теперь $\{u, v\} \in \widetilde{X}(G(t))$ и вершины $u$ и $v$ не принадлежат одному и тому же слою $V(t, h)$ дерева $t$. Без ограничения обшности можно считать $u \in V(t, h)$, 
$v \in V(t, h+1)$, где $0 \leqslant h \leqslant H(t)-1$. При этом возможны два случая, когда $(u, v)$ является либо дугой дерева $t$, либо дугой из множества $Y(t, v)$.

Допустим, что $(u, v)$ - дуга дерева $t$. Тогда $(\omega(u), \omega(v)) \in X(A t)$, а $\{\omega(u), \omega(v)\}-$ ребро максимального надграфа $G(A t)$ дерева $A t$, которое получается из дуги $(\omega(u), \omega(v))$, если ее лишить ориентации.

Допустим теперь, что $(u, v) \in Y(t, v)$. Обозначим через $u(v)$ вершину дерева $t$, из которой исходит дуга дерева $t$, входящая в вершину $v$. Из определения множества $Y(t, v)$ следует, что $u(v)<u$. Поэтому из определения отображения $\omega$ вытекает, что $\omega(u(v))<\omega(u)$, причем из равенства (16) видно, что вершины $\omega(u(v))$ и $\omega(u)$ принадлежат одному и тому же слою $V(A t, h)$ дерева $A t$. Поскольку $(\omega(u(v)), \omega(v))$ дуга дерева $A t$, то $(\omega(u), \omega(v))$ - дуга множества $Y(A t, \omega(v))$, а $\{\omega(u), \omega(v)\}$ - ребро максимального надграфа $G(A t)$ дерева $A t$. Таким образом, отображение $\omega$ удовлетворяет условию 2 определения 5. Аналогично доказывается, что отображение $\omega$ удовлетворяет условию 3 определения 5 , т.е. обратное отображение $\omega^{-1}$ множества вершин $V_{n}$ на множество вершин $V$ сохраняет смежность пар вершин максимального надграфа $G(A t)$ дерева $A t$. Отсюда следует утверждение леммы 2.

Лемма 3. Отображсение $А$ является взаимно однозначным отображснием множества $T(V, \alpha)$ на множество $T_{n}$.

ДоказАтельство. Докажем, что отображение $A$ является взаимно однозначным отображением. Допустим противное. Тогда сушествуют такие деревья $t_{1}, t_{2} \in T(V, \alpha)$, $t_{1} \neq t_{2}$, что $A t_{1}=A t_{2}=t$. При этом из определения отображения $A$ вытекает, что

$$
\Omega\left(X\left(t_{1}\right)\right)=\Omega\left(X\left(t_{2}\right)\right)
$$

Так как $t_{1} \neq t_{2}$, то, очевидно, что $X\left(t_{1}\right) \neq X\left(t_{2}\right)$, и, следовательно, $X\left(t_{1}\right) \backslash X\left(t_{2}\right) \neq \varnothing$. Рассмотрим дугу $(u, v) \in\left[X\left(t_{1}\right) \backslash X\left(t_{2}\right)\right]$. Из равенства (17) следует, что в множестве $X\left(t_{2}\right)$ имеется такая дуга $\left(u^{\prime}, v^{\prime}\right) \neq(u, v)$, что $\Omega\left(\left(u^{\prime}, v^{\prime}\right)\right)=\Omega((u, v))$. Однако это противоречит тому, что отображение $\Omega$ является взаимно однозначным. Следовательно, множество $A(T(V, \alpha))$ содержит столько же различных элементов, сколько элементов содержит множество $T(V, \alpha)$, т.е. $n^{n-2}$ различных элементов из множества $T_{n}$. Отсюда и вытекает, что $A(T(V, \alpha))=T_{n}$. Значит, отображение $A$ является взаимно однозначным отображением множества $T(V, \alpha)$ на множество $T_{n}$. Лемма 3 доказана.

Рассмотрим майеровскую функцию

$$
f_{i j}=\exp \left\{-\beta \Phi\left(\mathbf{r}_{i}-\mathbf{r}_{j}\right)\right\}-1
$$

и введем обозначения

$$
\begin{gathered}
(\mathbf{r})_{V}=\left\{\mathbf{r}_{v}, v \in V\right\} \\
(d \mathbf{r})_{V}=\prod_{v \in V} d \mathbf{r}_{v}
\end{gathered}
$$


Пусть $t \in T(V, \alpha)$. Обозначим

$$
\begin{gathered}
D\left(t,(\mathbf{r})_{V}\right)=\prod_{(u, v) \in X(t)} f_{u v} \prod_{\{\tilde{u}, \tilde{v}\} \in \tilde{X}^{\prime}(t)} \exp \left\{-\beta \Phi\left(\mathbf{r}_{\tilde{u}}-\mathbf{r}_{\tilde{v}}\right)\right\}, \\
I(t)=\int_{\left(\mathbf{R}^{\nu}\right)^{n-1}} D\left(t,(\mathbf{r})_{V}\right)(d \mathbf{r})_{V \backslash\{\alpha\}} .
\end{gathered}
$$

В статье [10] доказано, что для любого растушего дерева $t$ с множеством $V$ помеченных вершин и корнем $\alpha \in V$ несобственный интеграл $I(t)$ сходится в случае устойчивого и регулярного парного потенциала и не зависит от значения $\mathbf{r}_{\alpha}$. Так как неотрицательный потенциал является устойчивым, то отсюда вытекает, что несобственный интеграл $I(t)$ сходится в случае неотрицательного и регулярного парного потенциала.

В работе [3] было получено следуюшее представление коэффициентов $b_{n}(\beta)$ в случае устойчивого и регулярного парного потеншиала:

$$
b_{n}(\beta)=\frac{1}{n !} \sum_{t \in T_{n}} I(t), \quad n=2,3, \ldots
$$

Поскольку неотрицательный потенциал является устойчивым, то представление (23) коэффициентов $b_{n}(\beta)$ имеет место, в частности, в случае неотрицательного регулярного парного потенциала.

ЛЕмма 4. Если парный потенциал $\Phi(\mathbf{r})$ устойчивый и регулярный, то для любого множества вершин $V$ и при любом $\alpha \in V$ имеет место равенство

$$
\sum_{t \in T(V, \alpha)} I(t)=\sum_{t \in T_{n}} I(t)=n ! b_{n}(\beta),
$$

где $n=|V|$.

ДОКАЗАТЕЛЬСТвО. Так как парный потенциал $\Phi(\mathbf{r})$ устойчивый и регулярный, то интеграл $I(t)$ сходится для любого помеченного растушего дерева $t$. Из определения интеграла $I(t)$ следует, что для любых двух максимально изоморфных деревьев $t$ и $t^{\prime}$ имеет место равенство $I(t)=I\left(t^{\prime}\right)$. Введенное вьше отображение $A$ является по лемме 3 взаимно однозначным отображением множества деревьев $T(V, \alpha)$ на множество деревьев $T_{n}$. При этом по лемме 2 деревья $t \in T(V, \alpha)$ и $A t \in T_{n}$ максимально изоморфные и для них имеет место равенство

$$
I(t)=I(A t) .
$$

Отсюда вытекает, что

$$
\sum_{t \in T(V, \alpha)} I(t)=\sum_{t \in T(V, \alpha)} I(A t)=\sum_{t \in T_{n}} I(t) .
$$

Из соотношений (26) и (23) следует соотношение (24). Лемма 4 доказана. 
Рассмотрим растушее дерево $t$ с помеченными вершинами и корнем $\alpha(t)$. Обозначим через $M(t)=\{m\}$ такую совокупность натуральных чисел, что в слое вершин $V(t, m)$ дерева $t$ лишь одна вершина $v(t, m)$ не является висячей, причем в слое вершин $V(t, m)$ нет вершин, следующих за вершиной $v(t, m)$.

Пусть множество $M(t)$ непусто и $m \in M(t)$. Введем следуюшие обозначения: $t_{1}(t, m)$ - поддерево, которое получается из дерева $t$ удалением всех вершин, достижимых из вершины $v(t, m)$, за исключением вершины $v(t, m) ; t_{2}(t, m)$ - поддерево с корнем $v(t, m)$, получаюшееся из дерева $t$ удалением всех вершин, не достижимых из вершины $v(t, m)$. Очевидно, что дерево $t$ есть объединение [8] поддеревьев $t_{1}(t, m)$ и $t_{2}(t, m)$, т.е.

$$
t=t_{1}(t, m) \cup t_{2}(t, m)
$$

Из определения поддеревьев $t_{1}(t, m)$ и $t_{2}(t, m)$ следует $[7,10,11]$, что

$$
\begin{aligned}
X(t) & =X\left(t_{1}(t, m)\right) \cup X\left(t_{2}(t, m)\right), \\
G(t) & =G\left(t_{1}(t, m)\right) \cup G\left(t_{2}(t, m)\right), \\
\tilde{X}^{\prime}(t) & =\tilde{X}^{\prime}\left(t_{1}(t, m)\right) \cup \tilde{X}^{\prime}\left(t_{2}(t, m)\right) .
\end{aligned}
$$

Обозначим через $T(n \mid s)$ множество деревьев $t \in T_{n}$, удовлетворяющих условию $t_{1}(t, H(s))=s$, а через $V(t)$ - множество вершин дерева $t ; n(t)=|V(t)|$.

Каково бы ни было растушее помеченное дерево $s$ с корнем $\alpha=1$, удовлетворяюшее условию

$$
V(s) \subset V_{n},
$$

для всех деревьев $t$ из множества $T(n \mid s)$ выполняется соотношение $H(s) \in M(t)$. Кроме того, все деревья $t$ из множества $T(n \mid s)$ имеют одну и ту же вершину $v(t, H(s))$, которая однозначно определяется деревом $s$. Обозначим эту вершину $v(s)$.

Если $s \in T(V, 1)$, где $V \subset V_{n}$, то совокупность деревьев $\left\{t_{2}(t, H(s)): t \in T(n \mid s)\right\}$ совпадает с совокупностью деревьев $T\left(\left(V_{n} \backslash V\right) \cup\{v(s)\}, v(s)\right)$.

Лемма 5. Пусть парный потенииал $\Phi(\mathbf{r})$ является неотрицательным и регулярным, а растущее помеченное дерево $s$, имеющее своим корнем вериину $\alpha=1$, и число п удовлетворяют условию (30). Тогда имеет место соотношение

$$
\sum_{t \in T(n \mid s)} I(t)=I(s)(n-n(s)+1) ! b_{n-n(s)+1}(\beta) .
$$

ДокАЗАТЕЛьство. Из определения множества деревьев $T(n \mid s)$ и соотношений $(27),(29)$ следует, что для всякого дерева $t \in T(n \mid s)$ имеем

$$
\begin{gathered}
D\left(t,(\mathbf{r})_{n}\right)=D\left(s,(\mathbf{r})_{V(s)}\right) D\left(t_{2}(t, H(s)),(\mathbf{r})_{\left(V_{n} \backslash V(s)\right) \cup\{v(s)\}}\right), \\
I(t)=I(s) I\left(t_{2}(t, H(s))\right) .
\end{gathered}
$$


Из совпадения множества деревьев $\left\{t_{2}(t, H(s)): t \in T(n \mid s)\right\}$ с множеством деревьев $T\left(\left(V_{n} \backslash V(s)\right) \cup\{v(s)\}, v(s)\right)$ и из соотношения (33) следует, что

$$
\sum_{t \in T(n \mid s)} I(t)=I(s) \sum_{t \in T\left(\left(V_{n} \backslash V(s)\right) \cup\{v(s)\}, v(s)\right)} I(t) .
$$

Так как мощность множества $\left(V_{n} \backslash V(s)\right) \cup\{v(s)\}$ равна $n-n(s)+1$, то, применяя лемму 4 , из равенства (34) получаем равенство (31), что и требовалось доказать.

СлЕДСТвиЕ 1. Пусть парный потенциал $\Phi(\mathbf{r})$ является неотрицательным и регулярным, а число п и множество $V$ удовлетворяют условиям

$$
V \subset V_{n}, \quad 1 \in V .
$$

Тогда имеет место равенство

$$
\sum_{s \in T(V, 1)} \sum_{t \in T(n \mid s)} I(t)=|V| b_{|V|}(\beta)(n-|V|+1) ! b_{n-|V|+1}(\beta) .
$$

ДоКАЗАТЕЛЬСТво. По определению $T(V, 1)$ любое дерево $s$ из этого множества имеет своим множеством вершин множество $V$, а своим корнем - вершину $1 \in V$. При этом данные число $n$ и множество $V$ удовлетворяют условиям (35). Следовательно, любое дерево $s \in T(V, 1)$ удовлетворяет при данном $n$ условию (30). Применяя лемму 5 , получаем

$$
\sum_{s \in T(V, 1)} \sum_{t \in T(n \mid s)} I(t)=\sum_{s \in T(V, 1)} I(s)(n-|V|+1) ! b_{n-|V|+1}(\beta) .
$$

По лемме 4 имеем

$$
\sum_{s \in T(V, 1)} I(t)=|V| b_{|V|}(\beta) .
$$

Из (37) и (38) следует (36). Следствие 1 доказано.

Введем в рассмотрение множество деревьев $S(n, k)$, полагая

$$
S(n, k)=\bigcup_{V}^{\prime} \bigcup_{n, k} T(n \mid s),
$$

где $\bigcup_{n}^{\prime}$, означает суммирование по всем имеюшим мошность $k$ подмножествам $V$ множества $V_{n}$, удовлетворяюшим условиям (35).

ЛЕмма 6. Если парный потенциал $\Phi(\mathbf{r})$ является неотрицательныцм и регулярньц, то коэффичиенты $b_{n}(\beta)$ разложения (9) удовлетворяют неравенству

$$
n\left|b_{n}(\beta)\right| \geqslant k\left|b_{k}(\beta)\right|(n-k+1)\left|b_{n-k+1}(\beta)\right|,
$$

әде $2 \leqslant k<n$. 
ДокАЗАТЕЛЬСтво. Очевидно, что множество деревьев $S(n, k)$ входит в множество деревьев $T_{n}$. Поэтому множество $T_{n}$ можно представить в виде суммы двух непересекаюшихся подмножеств

$$
T_{n}=S(n, k) \cup\left(T_{n} \backslash S(n, k)\right) .
$$

Используя формулу (23) для коэффициентов $b_{n}(\beta)$, с помощью разложения (41) получаем, что

$$
b_{n}(\beta)=\frac{1}{n !} \sum_{t \in T_{n}} I(t)=\frac{1}{n !} \sum_{t \in S(n, k)} I(t)+\frac{1}{n !} \sum_{t \in\left(T_{n} \backslash S(n, k)\right)} I(t) .
$$

Так как парный потенциал $\Phi(\mathbf{r})$ неотрицательный, то все не равные нулю интегралы $I(t)$, входящие в равенство (42), имеют один и тот же знак. Поэтому из (42) следует, что

$$
\left|b_{n}(\beta)\right| \geqslant \frac{1}{n !}\left|\sum_{t \in S(n, k)} I(t)\right|=\frac{1}{n !} \sum_{V}^{\prime}\left|\sum_{n, k} \sum_{s \in T(V, 1)} I(t)\right|,
$$

где $\sum_{V}^{\prime}{ }_{n, k}$ означает суммирование по всем имеюшим мощность $k$ подмножествам $V$ множества $V_{n}$, удовлетворяющим условиям (35). Число таких подмножеств равно числу

$$
\left(\begin{array}{c}
n-1 \\
k-1
\end{array}\right)=\frac{(n-1) !}{(k-1) !(n-k) !} .
$$

Поэтому, применяя следствие 1 к правой части соотношений (43), после элементарных алгебраических преобразований получаем

$$
\begin{aligned}
n\left|b_{n}(\beta)\right| & \geqslant n(n !)^{-1}\left(\begin{array}{c}
n-1 \\
k-1
\end{array}\right)\left|k ! b_{k}(\beta)(n-k+1) ! b_{n-k+1}(\beta)\right|= \\
& =k\left|b_{k}(\beta)\right|(n-k+1)\left|b_{n-k+1}(\beta)\right| .
\end{aligned}
$$

Лемма 6 доказана.

СЛЕДСТВИЕ 2. Если парный потенциал $\Phi(\mathbf{r})$ является неотрицательным и регулярным, то при всех $k$, удовлетворяющих условию $2 \leqslant k<n$, имеет место неравенство

$$
n\left|b_{n}(\beta)\right| \geqslant\left\{k\left|b_{k}(\beta)\right|\right\}^{[(n-1) /(k-1)]}[r(n-1, k-1)+1]\left|b_{r(n-1, k-1)+1}(\beta)\right|,
$$

где $[a]$ означает иелую часть числа $a, r(n, k)$ - наименьший неотрицательньй вычет [12] числа п по модулю $k$.

ДокАЗАТЕЛьство. Применяя последовательно несколько раз лемму 6 , получаем

$$
\begin{aligned}
& n\left|b_{n}(\beta)\right| \geqslant k\left|b_{k}(\beta)\right|(n-k+1)\left|b_{n-k+1}(\beta)\right| \geqslant k^{2}\left|b_{k}(\beta)\right|^{2}(n-2 k+2)\left|b_{n-2 k+2}(\beta)\right| \geqslant \cdots \\
& \cdots \geqslant\left\{k\left|b_{k}(\beta)\right|\right\}^{[(n-1) /(k-1)]}[r(n-1, k-1)+1]\left|b_{r(n-1, k-1)+1}(\beta)\right| .
\end{aligned}
$$

Следствие 2 доказано. 
СЛЕДСТВИЕ 3. Если парный потенциал $\Phi(\mathbf{r})$ является неотрицательным и регулярным то коэффичиенты $b_{n}(\beta)$ разложсения (9) при любом $k \geqslant 2$ удовлетворяют неравенству

$$
\varlimsup_{n \rightarrow \infty}\left(n\left|b_{n}(\beta)\right|\right)^{-1 /(n-1)} \leqslant\left(k\left|b_{k}(\beta)\right|\right)^{-1 /(k-1)} .
$$

ДокАЗАТЕЛЬСТво. Из следствия 2 вытекает, что

$$
\begin{aligned}
& \left(n\left|b_{n}(\beta)\right|\right)^{-1 /(n-1)} \leqslant \\
& \quad \leqslant\left\{\left\{k\left|b_{k}(\beta)\right|\right\}^{[(n-1) /(k-1)]}(r(n-1, k-1)+1)\left|b_{r(n-1, k-1)+1}(\beta)\right|\right\}^{-1 /(n-1)} .
\end{aligned}
$$

Устремляя в этом неравенстве $n$ к бесконечности, получаем утверждение следствия 3 .

СЛЕДСТВИЕ 4. Если парный потенциал является неотрицательным и регулярньмм, то существует предел

$$
\lim _{n \rightarrow \infty}\left(n\left|b_{n}(\beta)\right|\right)^{-1 /(n-1)}
$$

ДокАЗАТЕЛЬСтво. Устремляя в неравенстве (47) величину $k$ к бесконечности, получаем

$$
\varlimsup_{n \rightarrow \infty}\left(n b_{n}(\beta)\right)^{-1 /(n-1)} \leqslant \varliminf_{k \rightarrow \infty}\left(k\left|b_{k}(\beta)\right|\right)^{-1 /(k-1)} .
$$

Из (50) следует сушествование предела (49). Следствие 4 доказано.

ДОКАЗАТЕЛЬСТВО ТЕОРЕМЫ. Из следствия 4 вытекает, что ряд в правой части (9) имеет радиус сходимости $R$, равньй пределу (49). Отсюда по следствию 3 следует, что при любом $k \geqslant 2$ величина $\left(k\left|b_{k}(\beta)\right|\right)^{-1 /(k-1)}$ является оценкой сверху радиуса сходимости $R$, т.е.

$$
R \leqslant\left(k\left|b_{k}(\beta)\right|\right)^{-1 /(k-1)} .
$$

Теорема доказана.

ЗАмечАниЕ 1 . Первый член $\left(2\left|b_{2}(\beta)\right|\right)^{-1}$ последовательности оценок (10) совпадает с оценкой Гренвельда

$$
R<[C(\beta)]^{-1}
$$

В самом деле, т.к. потенциал $\Phi(\mathbf{r})$ по условию неотрицательный и регулярный, то функция Майера $f(\mathbf{r})$ является неположительной и интегрируемой по пространству $\mathbf{R}^{\nu}$, поэтому

$$
2\left|b_{2}(\beta)\right|=\int_{\mathbf{R}^{\nu}}\left|f\left(\mathbf{r}_{1}-\mathbf{r}_{2}\right)\right| d \mathbf{r}_{2}=C(\beta) .
$$

Следовательно, оценка Гренвельда (52) является первым членом последовательности оценок (10). 
ЗАмечание 2. При $k>2$ оценка (51) лучше, чем оценка Гренвельда (52). Действительно, множество деревьев $T_{n}$ можно представить в виде суммы двух непересекающихся множеств:

$$
T_{n}=P_{n} \cup\left(T_{n} \backslash P_{n}\right),
$$

где $P_{n}=\{t\}-$ совокупность всех деревьев $t \in T_{n}$, являющихся направленными путями [8]. Очевидно, что мошность множества $P_{n}$ равна $(n-1)$ !.

Все деревья $t \in P_{n}$ максимально изоморфны дереву $t_{0} \in P_{n}$ с множеством вершин $V_{n}$ и множеством дуг $X\left(t_{0}\right)=\{(i, i+1), i=1,2, \ldots, n-1\}$, и для них имеет место равенство

$$
I(t)=I\left(t_{0}\right)=\int_{\left(\mathbf{R}^{\nu}\right)^{n-1}} \prod_{i=1}^{n-1} f_{i, i+1}(d \mathbf{r})_{1, n-1}=\left(\int_{\mathbf{R}^{\nu}} f(\mathbf{r}) d \mathbf{r}\right)^{n-1} .
$$

Отсюда вытекает, что

$$
\frac{1}{(n-1) !} \sum_{t \in P_{n}} I(t)=\frac{1}{(n-1) !}\left|P_{n}\right| I_{0}\left(t_{0}\right)=\left(\int_{\mathbf{R}^{\nu}} f(\mathbf{r}) d \mathbf{r}\right)^{n-1}
$$

Используя представление коэффициентов $b_{n}(\beta)$ формулой $(23)$, с помощью разложения (53) получаем

$$
n b_{n}(\beta)=\frac{1}{(n-1) !} \sum_{t \in P_{n}} I(t)+\frac{1}{(n-1) !} \sum_{t \in\left(T_{n} \backslash P_{n}\right)} I(t)
$$

Так как потенциал $\Phi(\mathbf{r})$ неотрицательный и регулярный, то все не равные нулю интегралы $I(t)$, входящие в равенство (56), имеют один и тот же знак, а среди деревьев, входящих в множество $T_{n} \backslash P_{n}$, найдутся такие деревья $t$, для которых интеграл $I(t)$ отличен от нуля. Поэтому из соотношения (56), используя равенство $(55)$, получаем

$$
n\left|b_{n}(\beta)\right|>\frac{1}{(n-1) !} \sum_{t \in P_{n}}|I(t)|=\left(\int_{\mathbf{R}^{\nu}}|f(\mathbf{r})| d \mathbf{r}\right)^{n-1}=[C(\beta)]^{n-1}
$$

откуда следует, что

$$
\left(k\left|b_{k}(\beta)\right|\right)^{-1 /(k-1)}<\frac{1}{C(\beta)}
$$

и, значит, оценка (51) при всех $k>2$ лучше оценки Гренвельда (52).

ПримеР. Пусть $\Phi(\mathbf{r})$ - потенциал парного взаимодействия частиц с твердыми сердцевинами, распределенных в трехмерном пространстве $\mathbf{R}^{3}$,

$$
\Phi(\mathbf{r})= \begin{cases}\infty, & |\mathbf{r}|<a \\ 0, & |\mathbf{r}| \geqslant a\end{cases}
$$

Очевидно, что в этом случае

$$
C(\beta)=\frac{4}{3} \pi a^{3}
$$


и оценка Гренвельда принимает вид

$$
R<\frac{1}{\frac{4}{3} \pi a^{3}}
$$

Вычислим оценку сверху $\left(3\left|b_{3}\right|\right)^{-1 / 2}$ радиуса сходимости $R$, являюшуюся вторым членом последовательности оценок (10). Очевидно, что множество $T_{3}$ состоит из двух направленных путей, входящих в множество $P_{3}$, и дерева $t^{\prime}$ с множеством дуг $X\left(t^{\prime}\right)=\{(1,2),(1,3)\}$. Для каждого дерева $t \in P_{3}$ по формуле (54) имеем

$$
I(t)=\left(\int_{\mathbf{R}^{3}} f(\mathbf{r}) d \mathbf{r}\right)^{2} .
$$

Вычисляя интеграл $I\left(t^{\prime}\right)$ по формулам (21) и (22), получаем

$$
I\left(t^{\prime}\right)=\int_{\left(\mathbf{R}^{3}\right)^{2}} f_{12} f_{13}\left(1+f_{23}\right) d \mathbf{r}_{2} d \mathbf{r}_{3}=\frac{17}{18} \pi^{2} a^{6} .
$$

Из формул (59) и (60) следует равенство

$$
3\left|b_{3}\right|=\frac{1}{2 !}\left[2 \frac{16}{9} \pi^{2} a^{6}+\frac{17}{18} \pi^{2} a^{6}\right]=\frac{9}{4} \pi^{2} a^{6} .
$$

Отсюда по формуле (51) получаем оценку

$$
R<3 b_{3}{ }^{-1 / 2}=\frac{2}{3 \pi a^{3}}=\frac{8}{9} \frac{1}{\frac{4}{3} \pi a^{3}},
$$

лучшую, чем оценка Гренвельда (52).

Улучшим оценку (61), воспользовавшись неравенством

$$
R<\left(4\left|b_{4}\right|\right)^{-1 / 3}
$$

вытекающим из теоремы этой статьи.

Множество $T_{4}$ содержит шесть направленных путей, принадлежащих множеству $P_{4}$, дерево $t_{1}$ с множеством дуг $X\left(t_{1}\right)=\{(1,2),(1.3),(3,4)\}$ и еще двумя максимально изоморфными ему деревьями, дерево $t_{2}$ с множеством дуг $X\left(t_{2}\right)=\{(1,2),(2,3),(2,4)\}$ и еше двумя максимально изоморфными ему деревьями, дерево $t_{3}$ с множеством дуг $X\left(t_{3}\right)=\{(1,2),(1,3),(2,3)\}$ и еше двумя максимально изоморфными ему деревьями и дерево $t_{4}$ с множеством дуг $X\left(t_{4}\right)=\{(1.2),(1,3),(1,4)\}$.

Для каждого дерева $t \in P_{4}$ по формуле (54) находим, что

$$
I(t)=\left(\int_{\mathbf{R}^{3}} f(\mathbf{r}) d \mathbf{r}\right)^{3}
$$


Вычисляя интегралы $I\left(t_{1}\right), I\left(t_{2}\right)$ и $I\left(t_{3}\right)$ по формулам $(21)$ и $(22)$, получаем

$$
\begin{aligned}
& I\left(t_{1}\right)=I\left(t_{2}\right)=-\frac{34}{27} \pi^{3} a^{9}, \\
& I\left(t_{3}\right)=-\frac{11923}{11340} \pi^{3} a^{9} .
\end{aligned}
$$

Так как для любых двух максимально изоморфных деревьев $t$ и $t^{\prime}$ имеет место равенство $I(t)=I\left(t^{\prime}\right)$, а $I\left(t_{4}\right)<0$, то из формул $(63)-(65)$ следует

$$
4\left|b_{4}\right|>\frac{1}{3 !} \pi^{3} a^{9}\left(\frac{64}{27}+3 \frac{34}{27}+3 \frac{11923}{11340}\right)=\frac{94243 \pi^{3} a^{9}}{22680} .
$$

Отсюда вытекает оценка

$$
R<\left(4\left|b_{4}\right|\right)^{-1 / 3}<\frac{\sqrt[3]{22680}}{\sqrt[3]{94243 \pi^{3} a^{9}}}<0.83 \cdot \frac{1}{\frac{4}{3} \pi a^{3}}
$$

что лучше как оценки Гренвельда (52), так и оценки (61).

Автор считает своим приятным долгом выразить благодарность И. И. Иванчику за постановку задачи и полезные обсуждения проблем статистической механики.

Работа поддержана Российским фондом фундаменальных исследований, грант № 98-01-01029.

\section{Список литературы}

[1] Д. Рюэль. Статистическая механика. Строгие результаты. М.: Мир, 1971.

[2] Дж. Майер, М. Гепперт-Майер. Статистическая механика. М.: Мир, 1980.

[3] Г. И. Калмыков. ТМФ. 1990. Т. 84. № 2. С. 279-289.

[4] Дж. Уленбек, Дж. Форд. Лекции по статистической механике. М.: Мир, 1965.

[5] J. Groeneveld. Phys. Lett. 1962. V. 3. № 1. P. 50-51.

[6] Г. И. Калмыков. Дискретная математика. 1992. Т. 4. Вып. 2 С. 66-73.

[7] Г. И. Калмыков. ТМФ. 1992. Т. 92. № 1. С. 139-149.

[8] Ф. Харари. Теория графов. М.: Мир, 1973.

[9] Ф.Я. Ветухновский. Графыы и сети. В сб.: Дискретная математика и математические вопросы кибернетики. Т. 1. Ред. С. В. Яблонский, О.Б. Лупанов. М.: Наука, 1974.

[10] Г. И. Калмыков. ТМФ. 1994. Т. 100. № 1. С. 44-58.

[11] Г. И. Калмыков. ТМФ. 1993. Т. 97. № 3. С. 452-458.

[12] И. М. Виноградов. Основы теории чисел. М.: Наука, 1981.

Поступила в редакцию 13.III.1998 г. 